\title{
Generator science and pragmatics: The "nuclear" in nuclear cardiology
}

\author{
William A. Van Decker, MD $^{\mathrm{a}}$ \\ a Division of Cardiology, Lewis Katz School of Medicine at Temple University, Philadelphia, PA
}

Received Jun 5, 2020; accepted Jun 8, 2020

doi: $10.1007 / \mathrm{s} 12350-020-02263-5$

\section{See related article, pp. 1728-1738}

In this issue of the Journal, Ahmadi et al. present a very technical science paper comparing a radionuclide generator performance parameter between the two commercially available $82 \mathrm{Sr} / 82 \mathrm{Rb}$ radionuclide generators used in PET Myocardial Perfusion Imaging with the daughter rubidium tracer. The studied parameter was daughter rubidium yield and percent yield efficiency (rubidium observed yield compared to theoretical idealized yield by radiochemistry calculated $\mathrm{Sr}$ generator decay formulas). Because the observed parameters are measured off the morning QA elutate and because each of the two generators use different approved proprietary closed infusion systems and recording QA outputs, a mathematical adjustment and curve fitting was performed to be able to compare the units in similar space. The manuscript would suggest a slight difference on this parameter between the two generators in a complex analysis. $^{1}$

This discussion creates an opportunity for the nuclear cardiology community to reflect on the medical radio isotopes we use and the importance of basic radiation physics and radiochemistry in moving the field forward with accessible safe isotopes of clinical importance.

It serves a purpose to place generator science in perspective with historical evolution and importance to clinical need. Primarily, all medical isotopes are produced either as by products of nuclear reactors (131 Iodine, most of medical 99 Molybdenum, etc.) or

Reprint requests: William A. Van Decker, MD, Division of Cardiology, Lewis Katz School of Medicine at Temple University, Philadelphia, PA ; william.vandecker@tuhs.temple.edu J Nucl Cardiol 2020;27:1739-42.

1071-3581/\$34.00

Copyright (C) 2020 American Society of Nuclear Cardiology. cyclotrons (PET agents, 82 Strontium, 201 thallium, etc.). Since the majority of hospitals/clinics will not have on site access to either and since the half-life decay of most medical isotopes are short, an intermediate local form of supply is necessary for accessible use. In 1920, a generator of $226 \mathrm{Ra} / 222 \mathrm{Rn}$ was the first patented nuclear generator. A generator works on the principle of efficiency separating a short-lived half-life usable radio isotope from a longer-lived half-life decaying parent radio isotope that has been loaded and bound onto a stable matrix column. A sterile non-pyrogenic-shielded environment becomes important in medical care. Gathering the daughter through the process called elution involves passing a solution (usually normal physiologic saline) through the system that will preferentially carry the desired daughter (and leave the parent behind) into an outflow solution called the elutate which can then be compounded as necessary and dose calibrated for patient delivery. Since the daughter is constantly being created by decay inside the generator it will come into a mathematical equilibrium (transient equilibrium as in the Moly-technetium generator or secular equilibrium as in $82 \mathrm{Sr} / 82 \mathrm{Rb}$ ) and appear within the lead to have the same observed half-life as the parent and be always available for elution. Simple in overview, the detailed science of the parent being radionuclide pure, the efficiency of getting as much usable daughter as possible from the system, and the ever present concern that unwanted parent will also come through into the elutate (bringing with it much longer half-life and unwanted radiation dosimetry exposure that gives no beneficial patient image) all make generator science an engineering feat to be impressed with and respected. ${ }^{2-4}$

The most common prototype nuclear generator in clinical nuclear medicine practice is the molybdenum (half-life 67 hours)-technetium (half-life 6 hours) generator ( alumina column) since it makes available 99m Technetium, still the majority used isotope across all fields of nuclear medicine. It has evolved and been in use for decades, making its practical debut in the 
approximate 1960s. A good portion of clinical sites obtain their technetium radio pharmaceuticals "unit dose" from a commercial radio pharmacy. This is made possible by the 6 hours half-life of technetium which allows it to be shipped within a reasonable geographic radius and still be within valid dosing and non-expired ranges when it reaches the end user. Commercial work is done for this type end user to maintain the generator and its radiation and sterility requirements. Additionally, all the NRC/FDA mandated QA elution generator testing (most especially that Molybdenum breakthrough testing has been performed and there is less than 0.15 microcuries of 99 Moly per millicurie of 99mTechnetium, note units) is done at the commercial radio pharmacy and all the kit compounding and kit QA and all the initial unit dosing measurement is done there as well. ${ }^{5,6}$ This delivers essentially ready to use radiopharmaceutical to the end user (with all the mandated QA done off site).

Generator-yield efficiency is the ratio of the observed produced daughter isotope of interest compared to the theoretic ideal amount that should be produced by parent decay and calculated by standard radio pharmacy physics of decay equations. Multiple factors may affect the observed yield of daughter and may include finite affinity of daughter to column and elutant (is some left behind in separation), mechanical entrapment of daughter in spaces of column, valent species of daughter, elution rates through generator not optimal, and the elution volume of the draw down not optimal. The usual quoted yield efficiency of the molytechnetium generator is quoted as usually greater than $90 \%$ with a package insert default of about $93 \%$. Having said that, generator science is evolutionary and some trials on varying column composition/elutant showed variations of $75 \%-95 \%$ efficiency. ${ }^{7}$ Suffice it to say that basic science investigations into methods to improve general radionuclide generator efficiency continue to this day. ${ }^{8}$ While observed yield of daughter can be performed during breakthrough testing for parent contamination of the initial elutate, more measurements of the parent are necessary at that time for this EXTREMELY important parameter of breakthrough safety.

$82 \mathrm{Sr} / 82 \mathrm{Rb}$ generators have some generic differences from moly-technetium generators. The daughter half-life is very short (about 75 seconds) compared to the parent 82 strontium (about 25.4 days). This sets up secular equilibrium and the short $\mathrm{Rb}$ half-life makes elution a continuous infusion process through closed in and out tubing with rapid re ingrowth recharging of the daughter fully in the generator (10-15 minutes) compared to the bolus solution elution in moly-technetium generator and the longer recharging daughter ingrowth post-elution of technetium. The $82 \mathrm{Sr} / 82 \mathrm{Rb}$ column is composed of hydrated tin oxide. The cyclotron production of strontium can be complex ${ }^{9}$ and carries with it a small amount of the radionuclide contaminant 85 strontium (half-life 64.8 days, energetic gamma) such that both $82 \mathrm{Sr}$ and $85 \mathrm{Sr}$ are loaded on the parent column and both can be "breakthrough" in the elutate. $\mathrm{Rb}$ generators have long been studied in an evolutionary manner with very early trial studies in 1976 suggesting yield efficiencies of $75-90$ plus $\%^{10}$ and ongoing investigations over the years which have included some of the current authors. ${ }^{11}$ The most prominent discussion of $82 \mathrm{Sr} / 82 \mathrm{Rb}$ generator performance parameters over the past decade has been discussion of occasional reports of $\mathrm{Rb}$ administration that included contaminant $82 \mathrm{Sr}$ and $85 \mathrm{Sr}$ breakthrough leading to recognizable downstream radiation burden. The first events of 2011 involved breakthrough QA testing and high volume of elutions and the newer events in 2018-2019 appear related to the use of Ringer's Lactate (containing Calcium) rather than normal saline for the elution. This has led to re-evaluation of action alerts, permissible breakthrough limits, volumes, QA and QA education on breakthrough testing, some box warnings on unintended Sr exposure beyond package insert limits, and reminders on elution solutions. The episodes are succinctly described in NRC Information Notice 2019-11, "Strontium-82/Rubidium-82 Generator Elution Events and Issues," Date December 23, 2019. ${ }^{12}$

The first approved commercial $82 \mathrm{Sr} / 82 \mathrm{Rb}$ generator was introduced by Bracco in 1989 (CardioGen-82) and a second approved commercial $82 \mathrm{Sr} / 82 \mathrm{Rb}$ generator has been available since about 2016 by Jubilant DraxImage ( Ruby-Fill). Each utilizes its own proprietary infusion system and its own proprietary QA process. The basic mechanics of the infusion system is outlined in the accompanying manuscript and other manuscripts ${ }^{13,14}$ involving a closed continuous infusion pump (peristaltic or perhaps otherwise) of normal saline tubed through the generator and then tubed out through a dose calibrator activity counter and then through a line with a diversion valve to either waste chamber or patient. Given proprietary nature, little is known about alternate chemical prep/conditioning of the column or physical geometry/ volume of exchanger in column between the two vendors. More specifics on the two generators can be found in their respective package inserts and each system needs to start with daily QA checks at its local site of use. CardioGen Stop Use shelf-life is at expiration limit of $17 \mathrm{~L}$ cumulative eluate volume or 42 days post generator calibration date or eluate $82 \mathrm{Sr}$ level of 0.01 microcuries per millicurie $82 \mathrm{Rb}$ or eluate $85 \mathrm{Sr}$ level of 0.1 microcuries per millicurie $82 \mathrm{Rb}$. Ruby-Fill Stop Use shelf-life includes the same NRC/FDA mandated limits of $82 \mathrm{Sr}$ and $85 \mathrm{Sr}$ described above or $30 \mathrm{~L}$ for the 
generator's cumulative eluate volume, or 60 days post manufacturing expiration date. Each vendor has their own specific action limits (volumes and $82 \mathrm{Sr} / 85 \mathrm{Sr}$ findings) prior to Stop use that mandate more than once a day QA testing. ${ }^{15,16}$

Each vendor's system approved daily QA is different in process to get to daily calibration/breakthrough testing necessitating mathematical calculations with some assumptions to create a conversion factor to a common unit for yield comparison in this associated study. The CardioGen 82 system tests a $50-\mathrm{mL}$ elution at a flow rate of $50 \mathrm{~mL} \cdot \mathrm{min}$ and the Ruby-Fill system tests a $35 \mathrm{~mL}$ elution at $20 \mathrm{~mL} \cdot \mathrm{min}$. CardioGen 82 reports $82 \mathrm{Rb}$ activity as "dose calibrated maximum" and the Ruby- Fill generator system reports $82 \mathrm{Rb}$ activity as "integrated total activity", Mathematic correction formulas were developed to convert "dose calibrated maximum Rb activity" of CardioGen 82 to "integrated total $\mathrm{Rb}$ activity estimate" in order to compare with Ruby-Fill reported yield. Results using these units and calculations suggested slightly better $\mathrm{Rb}$ yield efficiency of Ruby-Fill at $95 \pm 4 \%$ vs CardioGen 82 at $88 \pm 4 \%$. The mathematical comparison "conversion/corrected", modeling will without doubt "generate" much discussion in the science and technical and industry circles and likely lead to further explanations/criticisms in the future. However, it should be re assuring to clinical authorized users that both approved systems appear to have clinically relevant $\mathrm{Rb}$ yields, relevant approved ways to dose patient, and relevant approved processes to check mandated breakthrough testing.

Moreover, nuclear cardiology authorized users have proven repeatedly over the decades to be intellectually adept at integrating large amounts of science and data to decide between many available choices of SPECT and PET radiopharmaceuticals, camera acquisition systems, software quantification packages, etc., that will best serve their local environment and their patients. They well recognize that many parameters/characteristics will need to be weighed in the use of generators. In addition to yield, consideration of varying degrees needs to be given to 1. parent radionuclide breakthrough assessment for safety considerations, 2. ease and simplicity of use, 3. patient radiation dosimetry and dosimetry to staff, 4 . cost efficiency, 5. perceivable or not image quality changes based on reliable delivered count statistics, 6 . stability of generator during shelf-life and stability from delivered generator to delivered generator, 7. comments/ approvals by FDA and NRC, 8. vendor service support for their generator, and 9. availability. As usual in global assessments, the key will be understanding the science, understanding the trade-offs, making local decisions that work, and constantly reassessing the patient clinical/clinical outcome impact that is the North Star.
An important "by-product" of the discussion on a performance parameter for this specific non-reactorbased generator is the opportunity to step back and recognize the importance of hard nuclear science and its evolution to date. The technical, physics, and radiochemistry principles that make nuclear cardiology images possible and so clinically helpful to our patients when matched with good clinical judgment is a marvel to cherish. We all look forward to pragmatic advancements in nuclear science and nuclear imaging science that will create opportunities for continuing improvements in the delivery of seamless quality cardiovascular care.

\section{References}

1. Ahmadi A, Klein R, Lewin H, Beanlands R, deKemp R (2020) Rubidium-82 generator yield and efficiency for PET perfusion imaging: Comparison of two clinical systems. JNC. https://doi.org/ 10.1007/s12350-020-02200-6

2. Saha G (1992) Radiation generators. Chapter 5: Fundamentals of nuclear pharmacy. Springer, New York

3. Cherry S, Phelps M (2012) Radionuclide generator. Physics in nuclear medicine Fourth Edition. Elsevier, Amsterdam

4. Philips D (1996) Radionuclide generator systems for nuclear medicine. Continuing education for nuclear pharmacists and nuclear medicine professionals. Pharmacy Continuing Education. Univ. of New Mexico

5. Radioisotopes in Medicine (2020) World Nuclear Association. h ttps://www.world-nuclear/information-library/

6. TechneLite Package Insert. Lantheus

7. Vucina J (2001) Elution efficiency of Mo-99/Tc-99m generators. Phys Chem Technol 2:125-130

8. Le V, Zoe P, Minh K, Le V, Le N (2014) Methods of increasing the performance of radionuclide generators used in nuclear medicine: Daughter nuclide build-up optimization, elutionpurification-concentration integration, and effective control of radionuclide purity. Molecules 19:7714-7756

9. Tatsuo I, Hermanne A, Ditroi F, Szucs Z, Mahunka I, Tarkanyl F (2002) Re-measurement of the excitation function of the $85 \mathrm{Rb}$ (p, 4n) $82 \mathrm{Sr}$ nuclear reaction near the threshold: Relevance to the production of a $82 \mathrm{Sr}(82 \mathrm{Rb})$ generator system with a medium energy cyclotron. J Nucl Sci Technol 2:1310-1313

10. Yano Y, Chu T, Budinger T, Grant P, Ogard A, Barnes J, O’Brien H, Hoop B (1977) Rubidium generators for imaging studies. J Nucl Med. 18:46-50

11. Alvarez-Diez T, deKemp R, Beanlands R, Vincent J (2019) Manufacture of strontium 82/rubidium 82 generators and quality control of rubidium 82 chloride for myocardial perfusion imaging in patients using Positron emission tomography. Appl Radiat Isotopes 1999(50):1015-1023

12. NRC Information Notice (2019-11) Strontium - 82/rubidium 82 generator elution events and issues. https://www.nrc.gov. Accessed 23 Dec 2019

13. Dash A, Chakravarty R (2019) Radionuclide generators: the prospect of availing PET radiotracers to meet current clinical needs and future research demands. Am J Nucl Med Mol Imaging 9:3066

14. Yoshinaga K, Klein R, Tamaki N (2010) Generator-produced rubidium 82 positron emission tomography myocardial perfusion 
imaging: From basic aspects to clinical applications. J Cardiol

55:163-173

15. CardioGen 82 Package Insert. Bracco

16. Ruby-Fill Package Insert Jubilant DraxImage
Publisher's Note Springer Nature remains neutral with regard to jurisdictional claims in published maps and institutional affiliations. 